Article

\title{
Anti-Congestion Route Planning Scheme Based on Dijkstra Algorithm for Automatic Valet Parking System
}

\author{
Luyang $\mathrm{Yu} *$ (D), Haobin Jiang and Lei Hua \\ Department of Vehicle Engineering, Jiangsu University, Zhenjiang 212013, China; jianghb@ujs.edu.cn (H.J.); \\ hualei@ujs.edu.cn (L.H.) \\ * Correspondence: 15805295910@163.com; Tel.: +86-158-0529-5910
}

Received: 14 October 2019; Accepted: 19 November 2019; Published: 21 November 2019

Featured Application: This anti-congestion route planning scheme will be used in the automatic valet parking area. It will have outstanding performance in the combination technology of $\mathrm{V} 2 \mathrm{X}$ environment and driverless technology.

\begin{abstract}
Based on the Dijkstra algorithm, with the parking parameters in the static state, the shortest route to each parking space of the parking lot without dynamic influence factors can be calculated. In the new technology background of the combination of the V2X environment and driverless technology, the dynamic influence factors, for example, the lanes occupancy situation caused by parking, can be considered to improve the shortest route with the new scheme in this paper. Then the final route that costs the least time to reach each parking space will be calculated. This is very important for the development of the intelligent transportation system in the parking lot environment.
\end{abstract}

Keywords: automatic valet parking; route plan; Dijkstra algorithm; ITS; V2X

\section{Introduction}

Valet parking technology is a service that can help people park their vehicles without any drivers' operation [1,2]. It was born based on automatic parking [3,4], and combines the advantages of automatic parking technology and driverless technology [5-8]. The user only needs to drive the vehicle to the designated drop-off area of the parking lot. After getting off the vehicle, the vehicle will go to the designated parking space for parking. People do not need to find the parking space on their own in the complex parking lot environment. The technology also can solve the parking problem for some drivers if they have trouble parking, because the vehicle is controlled by the system. The parking lot does not need to prepare much driving space for drivers with poor driving skills, because the driving and parking processes are all controlled by the system, so more parking spaces can be arranged in the same area.

In the existing intelligent parking lot guidance systems, vehicles are all controlled by human drivers, so the condition of the parking lot cannot be predicted accurately. It is very hard to plan the route line based on real-time conditions. Road congestion is likely to occur when the traffic volume is large.

In our research, we used the Dijkstra algorithm as a basic tool [9-12]. It is a kind of shortest path algorithm. This algorithm has been used in many areas, such as the pathfinding applications designed by Balado, the distribution network optimization problem solved by Binh, or the fuel economy area studied by $\mathrm{Xu}$ [13-15]. Shakhovska created a conceptual model of a tourists' guide system [16]. They took the duration of switching traffic lights into account and did further research 
on time dimension based on the Dijkstra algorithm. In fact, many researchers have made the similar attempt in the parking lot guidance system, such as a route planning scheme for a parking lot designed by Ge et al. or a parking guidance algorithm proposed by Yuan [17,18].

Their researches saved a lot of time for vehicle costs on the road, but the uncertain factors of human drivers will bring many errors into the system. They will not be suitable for the automatic valet parking system.

In the automatic valet parking system, the parking lot is implemented in the V2X environment [19-21]. In the V2X environment, vehicles can exchange information with other vehicles or infrastructures based on vehicle networking. Also, all the vehicles in the parking lot are all controlled by the base station. Real-time road information in the parking lot, such as the vehicle's driving route, parking time, etc., can be transmitted through the communication network to the base station. The information is used for the parking lot control system to perform the calculation of the parking space allocation and route planning of the subsequent vehicles [22].

The combination of the V2X environment and driverless technology is a big breakthrough in valet parking technology. It has been a great help in making sure of the real-time condition and predicting future conditions in the parking lot. Based on the Dijkstra algorithm and combined with the dynamic influence factors of the valet parking lot, this paper designed a route planning scheme to reach each parking space in the shortest time for an automatic valet parking lot.

The contributions of this paper are described as follows:

1. The combination of the V2X environment and driverless technology has been a great help in making sure of the real-time condition and predicting future conditions in the parking lot. This paper designed a method to transform the dynamic influence factors in the parking lot into data based on the new technology for further calculation.

2. Based on the obtainment of the high-precise data in the new technology environment, this paper designed a route planning scheme to reach each parking space with the shortest time. This scheme is suitable for a driverless parking lot. It has been a great help in reducing the congestion of the automatic valet parking lot.

\section{Research Status}

In the middle of the nineteenth century, the first mechanized parking system was based on the vertical Ferris wheel, which effectively solved the problem of stacking vehicles in limited space. Since then, the same system has been used extensively in the United States, Asia and Europe [23].

In 2006, Beinhaker et al. designed a routing system. This system can forecast traffic conditions to some extent, but the vehicles are all driven by people, so the accurate driving time for each vehicle cannot be calculated.

In 2011, Liu et al. designed a model of the large parking lots guidance information system. It mainly uses the ant colony optimization to design the paths for parking space guidance. Ant colony optimization is a good way to find the shortest path from the start point to the end. However, the algorithm only considers the distance of the route. There is a great possibility that vehicles will line up in the same short way and wait for a long time [24].

Wang et al. presented a new system for intelligent navigation inside a parking lot in 2015 [25]. After modeling the parking lot with a time-varying graph, the proposed system applies a time-varying shortest path algorithm and dynamically tunes arc transit times based on planned vehicle routes as well as traffic flow sensor data. The proposed system showed an improvement of about $40 \%$ in travel time when compared with a system that only applies the conventional Dijkstra algorithm. But the differences in driving skills of human drivers bring a lot of errors to the system. It will not be a good choice for a driverless parking lot.

In early 2015, the high-tech Internet company AIpark began to develop intelligent parking systems [26,27]. Under the intelligent parking lot control system developed by the company, the driver only needs to drive into the parking lot. The parking lot can automatically identify the license plate, 
guide the car to the empty parking space and plan the route. The technology solves the parking problem to some extent [28-31]. It is a relatively complete model of the intelligent parking guidance system. However, since the vehicle is driven by the driver, the intelligent parking system cannot control the vehicles' speed and the actual driving route. It makes the guiding route planning of the following vehicles difficult.

On 14 September 2018, the automatic valet parking technology jointly developed by Daimler and Bosch was exhibited in Beijing [32]. In the underground parking lot of the Mercedes-Benz passenger car China R\&D center, two Mercedes-Benz E63s were used for valet parking operation at the same time. The two-lane dual-vehicle synchronous valet parking process was successfully realized. After the vehicle enters the parking lot, vehicle control is handed over to the parking lot [33,34]. As shown in Figure 1, single-line light detection and ranging systems (Lidars) are placed in the parking lot. They are mainly used to sense the accessible areas and obstacles in the parking lot [35], so as to control the vehicle for emergency braking or winding in time.

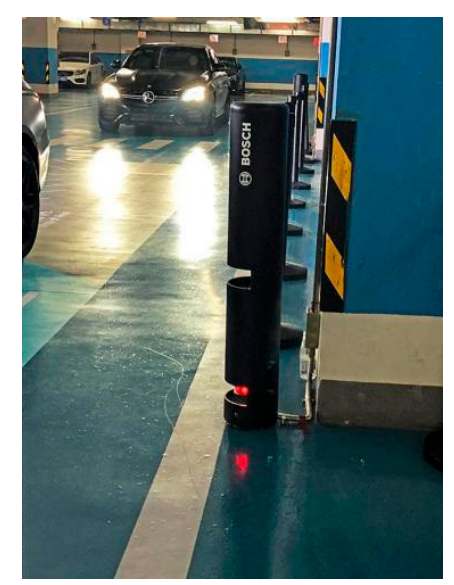

Figure 1. Industrial grade single-line laser radar.

The appearance of this technology can make the vehicles in the parking lot completely controlled by the base station. It reduces the occurrence of unexpected situations. However, in terms of route planning, the system cannot adjust the route according to the real-time road conditions in the parking lot, and it is prone to occur congestion when facing large traffic.

Therefore, it is meaningful to avoid congested roads in the process of route planning. In the new technology background of the combination of the V2X environment and driverless technology, we did research on how to design the route to reach each parking space with the least time based on the real-time working conditions in the parking lot.

\section{Dijkstra Algorithm}

The Dijkstra algorithm was proposed by Dutch computer scientists in 1959 [14,15]. It is a kind of shortest path algorithm used to calculate the shortest path from one node to another. The main idea of the algorithm is similar to the breath-first search.

\subsection{Algorithm Principle}

Divide the vertex set $V$ into two groups:

(1) $S$ : The set of vertices that have been determined (initially only contains the origin point $V_{0}$ )

(2) $\quad V-S=T$ : set of vertices that have not yet been determined

Add the vertices in $T$ to $S$ in ascending order to ensure:

(1) The length from $V_{0}$ to the other vertices in the $S$ is not longer than the shortest route length from $V_{0}$ to any vertices in $T$. 
(2) Each vertex corresponds to a distance value

Vertices in $S$ : the length from $V_{0}$ to the vertex

Vertices in $T$ : the shortest route length from $V_{0}$ to the vertex that only include the vertices in $S$ as the middle vertices

\subsection{Algorithm Demonstration}

(1) Point $\mathrm{A}$ is the starting point, allocated in the set $S$, and the remaining points are allocated in the set $T$.

(2) Initializing, except for the starting point A, the weights of all nodes are set to infinity. As shown in Figure 2a.

(3) Updating the distance of the A's adjacent point. Since the weight of AB is 2, the value of Distance (B) is updated to 2. Similarly, the value of Distance (D) is updated to 1. As shown in Figure 2b.

(4) Moving the point with the minimum weight to the set $S$. The value of Distance (D) is 1 , so move the D point to the set $S$.

(5) Calculating the weights of the adjacent points, starting from point $\mathrm{D}$. The weight is equal to the distance of $\mathrm{AD}$ plus the weights of point $\mathrm{D}$ to its adjacent point. Distance $(\mathrm{C})=1+2=3$, Distance $(\mathrm{E})=1+2=3$, Distance $(\mathrm{F})=1+8=9$, Distance $(\mathrm{G})=1+4=5$. As shown in Figure 2c.

(6) In all current nodes, node B with the minimum weight (Distance $(B)=2$ ) is moved to set $S$ and the weights of its adjacent points are updated.

(7) And so on, each time the node's weights are updated, the node with the minimum weight is moved to set $S$, and the node with the minimum weight is used as the starting point to update the weights of its adjacent nodes until all the nodes are moved from set $T$ to set $S$. After that, the current value of each node is the minimum weight from point $A$ to itself, as shown in Figure $2 \mathrm{~d}$.

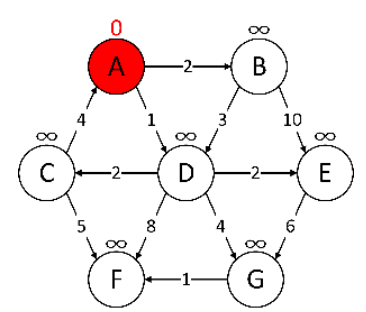

(a) Initializing

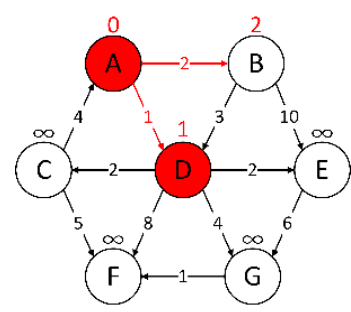

(b) Searching and updating

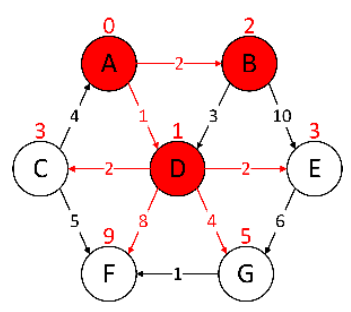

(c) Continue searching

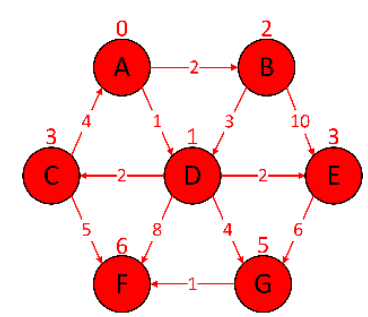

(d) Final result

Figure 2. Dijkstra algorithm.

\section{Anti-Congestion Route Planning Based on Dijkstra Algorithm for Valet Parking System}

In the valet parking scenario, the vehicle can obtain real-time conditions in the parking lot from the base station [36]. Based on the Dijkstra algorithm, this scheme combines the parking lot map information and the dynamic influence factors in the parking lot to plan the route to each parking space which costs the least time.

The specific program steps are as follows:

(1) Obtaining the parking space, road information map of the parking lot and the prescribed speed of the vehicle in the parking lot.

(2) According to the signal transmitted by the parking space geomagnetic sensor [37], the free parking space is confirmed. The parking lot entrance, the free parking space and the crossroads inside the parking lot are set to be nodes. Then connect them to their adjacent nodes to construct an undirected graph with weights. 
(3) Based on the undirected graph with weights, the adjacency matrix composed of the weights between nodes is shown in Equation (1). The value of the diagonal of the matrix is 0 . The values of adjacent nodes are the distances between them, and the remaining values are endless.

$$
\left[\begin{array}{ccccc}
0 & D_{12} & D_{13} & \ldots & D_{1 n} \\
D_{21} & 0 & D_{23} & \ldots & D_{2 n} \\
D_{31} & D_{32} & 0 & \ldots & D_{3 n} \\
\ldots & \ldots & \ldots & \ldots & \ldots \\
D_{n 1} & D_{n 2} & D_{n 3} & \ldots & 0
\end{array}\right]
$$

(4) Using this matrix as the data source of the Dijkstra algorithm, and calculating the shortest distance and the shortest route from the entrance to each parking space.

(5) In the V2X environment of the valet parking lot, real-time dynamic influence factors (remaining parking time of the vehicle, the position of the moving vehicle and its driving route) can be obtained and then take them into consideration.

(6) After the vehicle owner finishes the selection of the parking space, checking the driving route to the parking space planned in step 4. Making sure that if there is any dynamic influence factor mentioned in the route in step 5.

(7) Updating the corresponding matrix values between the nodes lie in the two ends of the road which contains the dynamic influencing factors, and then re-calculating with the Dijkstra algorithm.

If there is a car parking in the route, the corresponding matrix value between the two nodes is updated as follows:

(1) Calculating the time $t_{0}$ that the current vehicle costs to reach the occupied parking space based on the distance $d_{1}$ from the starting point to the parking space.

(2) Comparing the $t_{0}$ with the parking space's occupied time $t_{1}$. If $t_{0}>t_{1}$, it means that when the current vehicle arrives at the occupied parking space, the parking vehicle has completed parking, and the corresponding matrix value between the two nodes does not need to change. If $t_{0}<t_{1}$, it means that when the current vehicle arrives at the occupied parking space, the parking vehicle does not complete parking, then the corresponding matrix value between the two nodes is calculated according to Equation (2).

$$
D=\left(t_{1}-t_{0}\right) \cdot v_{0}+D_{0}
$$

If there is an allocated parking space in the route and the vehicle pairing with the parking space is driving toward the parking space, the corresponding matrix value between the two nodes is updated as follows:

(1) Calculating the time $t_{0}$ that the current vehicle costs to reach the allocated parking space based on the distance $d_{1}$ from the starting point to the parking space.

(2) Calculating the time $t_{2}$ that the vehicle pairing with the parking space costs to reach the parking space based on the distance $d_{2}$ from the current location of the vehicle to the parking space.

(3) Comparing $t_{0}$ with $t_{2}$. If $t_{0}<t_{2}$, it means that when the current vehicle arrives at the allocated parking space, the vehicle pairing with the parking space has not arrived at the allocated parking space, and the corresponding matrix value between the two nodes does not need to change. If $t_{0}>t_{2}$, it means that when the current vehicle arrives at the allocated parking space, the vehicle pairing with the parking space has reached the parking space and starts automatic parking, then the corresponding matrix value between the two nodes is calculated according to Equation (3).

$$
D=\left[50-\left(t_{0}-t_{2}\right)\right] \cdot v_{0}+D_{0}
$$


The 50 in the formula means that the most automatic parking systems on the market need to complete the automatic parking in the standard parking space is about $50 \mathrm{~s}$. This is because, when the vehicle in the parking lot does not reach the parking space, the automatic parking system cannot finish the identification of the parking space, the route planning and other processes, so take we $50 \mathrm{~s}$ as an estimate of the time required for parking.

(8) After the matrix value update is completed, the value of the new matrix is brought into the Dijkstra algorithm for calculation, and the new route is obtained from the starting point to reach each parking space.

(9) Extracting the driving route of the corresponding parking space in the new calculated route result. If the driving route is unchanged or the new dynamic influencing factors do not appear in the new route, the route is selected as the optimal route; if the driving route changes and there is an unconsidered dynamic influencing factor in the new route, the weight of the corresponding road is optimized by the method of step 7 until the driving route is unchanged or there is not any dynamic influencing factor appearing in the new route. Selecting the current route as the optimal route.

The whole process is shown in Figure 3.

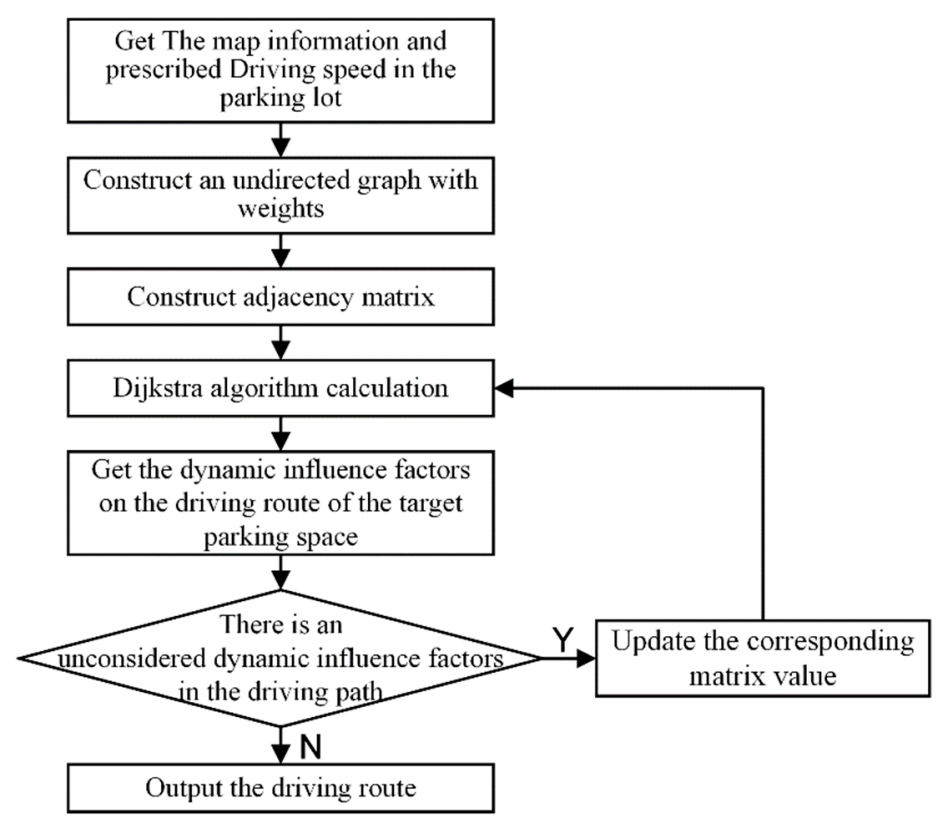

Figure 3. Route planning process.

\section{Simulation}

\subsection{Simulation Example}

(1) The target parking lot which was selected as the simulation prototype is shown in Figure 4. As shown in Figure 5, We have redesigned the parking spaces and roads in the target parking lot according to the Engineering Construction Industry Standard JGJ 100-98. 


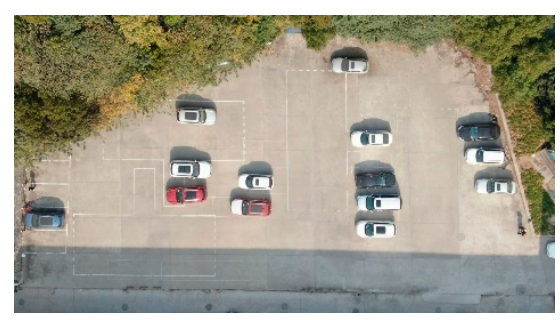

Figure 4. Target parking lot.

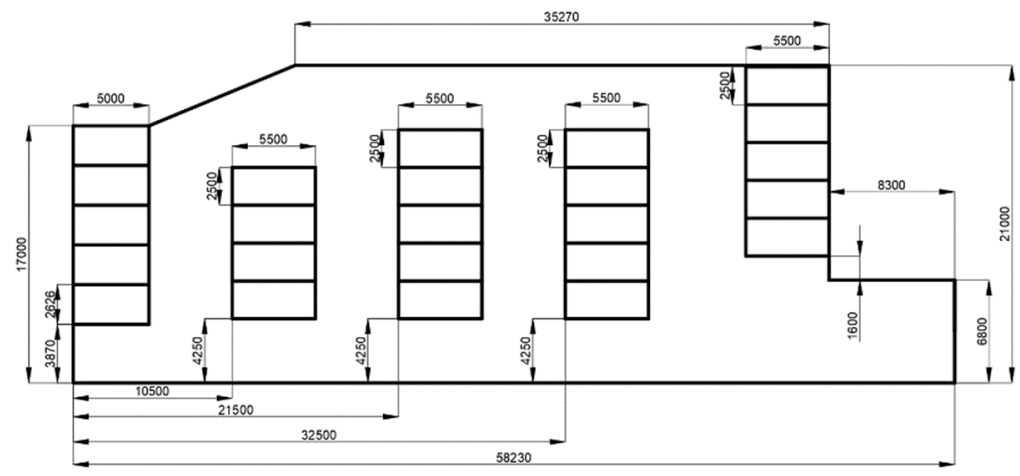

Figure 5. Parking lot map.

(2) We have extracted the parking spaces and lanes in Figure 5 as the frame of Figure 6. As shown in Figure 6, we have set a working condition in the parking lot. The solid circles represent the occupied parking spaces and the hollow circles represent the parking lot entrance, free parking spaces, and crossroads.

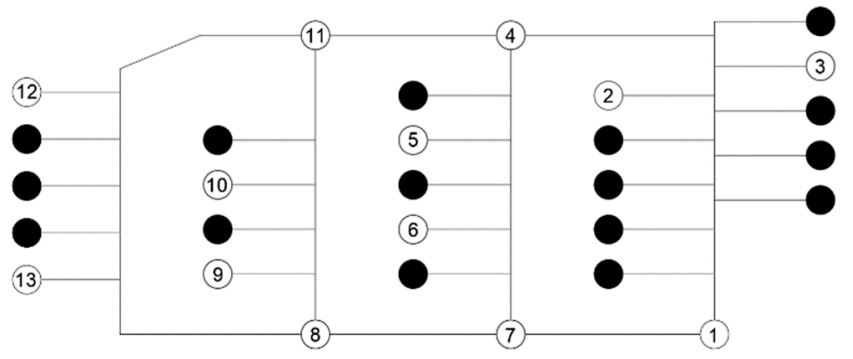

Figure 6. Parking lot occupancy.

(3) We have set the hollow circles in Figure 6 as nodes. Then we connected them to their adjacent nodes to construct an undirected graph with weights, as shown in Figure 7.

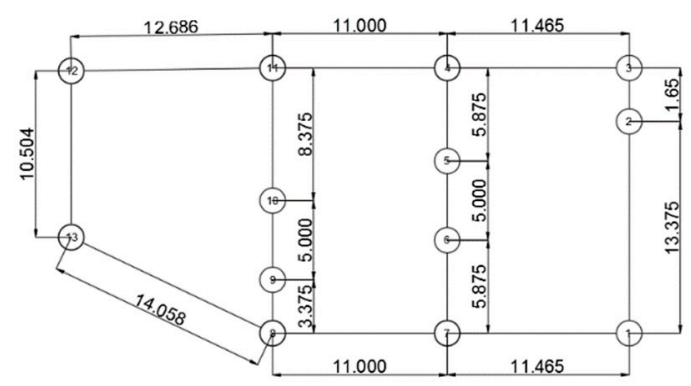

Figure 7. Undirected graph with weights. 
Based on the undirected graph with weights, the adjacency matrix composed of the weights between the nodes is shown in Equation (4).

$$
\left[\begin{array}{cccccccccccccc}
0 & 13.375 & \infty & \infty & \infty & \infty & 11.465 & \infty & \infty & \infty & \infty & \infty & \infty \\
13.375 & 0 & 1.65 & \infty & \infty & \infty & \infty & \infty & \infty & \infty & \infty & \infty & \infty & \infty \\
\infty & 1.65 & 0 & 13.19 & \infty & \infty & \infty & \infty & \infty & \infty & \infty & \infty & \infty \\
\infty & \infty & 13.19 & 0 & 5.875 & \infty & \infty & \infty & \infty & \infty & 11 & \infty & \infty & \infty \\
\infty & \infty & \infty & 5.875 & 0 & 5 & \infty & \infty & \infty & \infty & \infty & \infty & \infty \\
\infty & \infty & \infty & \infty & 5 & 0 & 5.875 & \infty & \infty & \infty & \infty & \infty & \infty \\
11.465 & \infty & \infty & \infty & \infty & 5.875 & 0 & 11 & \infty & \infty & \infty & \infty & \infty \\
\infty & \infty & \infty & \infty & \infty & \infty & 11 & 0 & 3.375 & \infty & \infty & \infty & 14.058 \\
\infty & \infty & \infty & \infty & \infty & \infty & \infty & 3.375 & 0 & 5 & \infty & \infty & \infty \\
\infty & \infty & \infty & \infty & \infty & \infty & \infty & \infty & 5 & 0 & 8.375 & \infty & \infty \\
\infty & \infty & \infty & 11 & \infty & \infty & \infty & \infty & \infty & 8.375 & 0 & 12.656 & \infty \\
\infty & \infty & \infty & \infty & \infty & \infty & \infty & \infty & \infty & \infty & 12.686 & 0 & 10.054 \\
\infty & \infty & \infty & \infty & \infty & \infty & \infty & 14.058 & \infty & \infty & \infty & 10.054 & 0
\end{array}\right]
$$

(4) Using this matrix as the data source of the Dijkstra algorithm, and calculating the shortest distance and the shortest route from the entrance to each parking space. The result is shown in Table 1.

Table 1. Shortest route and distance from the starting point to each parking space.

\begin{tabular}{ccc}
\hline Parking Space Number & Route & Distance/m \\
\hline Parking Space 2 & $1 \rightarrow 2$ & 13.375 \\
Parking Space 3 & $1 \rightarrow 2 \rightarrow 3$ & 15.025 \\
Parking Space 5 & $1 \rightarrow 7 \rightarrow 6 \rightarrow 5$ & 22.340 \\
Parking Space 6 & $1 \rightarrow 7 \rightarrow 6$ & 17.340 \\
Parking Space 9 & $1 \rightarrow 7 \rightarrow 8 \rightarrow 9$ & 25.840 \\
Parking Space 10 & $1 \rightarrow 7 \rightarrow 8 \rightarrow 9 \rightarrow 10$ & 30.840 \\
Parking Space 12 & $1 \rightarrow 7 \rightarrow 8 \rightarrow 13 \rightarrow 12$ & 47.027 \\
Parking Space 13 & $1 \rightarrow 7 \rightarrow 8 \rightarrow 13$ & 36.523 \\
\hline
\end{tabular}

(5) Setting the dynamic influence factors in the parking lot (the remaining parking time of the vehicle during parking, the moving vehicles' current position, and their driving routes). The test vehicle speed is set to $5 \mathrm{~km} / \mathrm{h}$, which not only ensures the safety of driving in the parking lot, but also satisfies the vehicle's recognition ability for surrounding parking spaces [38].

Figure 8 means that there are two cars going on automatic parking, the remaining time is $45 \mathrm{~s}$ and $50 \mathrm{~s}$, and there is a car in the parking lot, driving to follow the red line to the parking space pointed by the red arrow to start automatic parking.

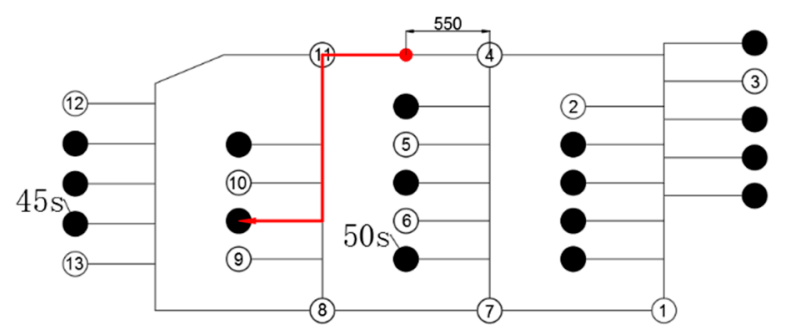

Figure 8. Real-time condition of the parking lot.

(6) Selecting the No. 6 node as the target parking space, and the driving route of the No. 6 parking space is $1 \rightarrow 7 \rightarrow 6$.

(7) There is a dynamic influence factor in the driving route of No. 6 parking space. It is a parking space being used for automatic parking, and the required time is $50 \mathrm{~s}$. It means that the lane will 
be occupied for $50 \mathrm{~s}$, and the weight of the corresponding road in the matrix needs to be corrected. As shown in Equation (5).

$$
\left[\begin{array}{ccccccccccccccc}
0 & 13.375 & \infty & \infty & \infty & \infty & 11.465 & \infty & \infty & \infty & \infty & \infty & \infty \\
13.375 & 0 & 1.65 & \infty & \infty & \infty & \infty & \infty & \infty & \infty & \infty & \infty & \infty & \infty \\
\infty & 1.65 & 0 & 13.19 & \infty & \infty & \infty & \infty & \infty & \infty & \infty & \infty & \infty \\
\infty & \infty & 13.19 & 0 & 5.875 & \infty & \infty & \infty & \infty & \infty & 11 & \infty & \infty \\
\infty & \infty & \infty & 5.875 & 0 & 5 & \infty & \infty & \infty & \infty & \infty & \infty & \infty \\
\infty & \infty & \infty & \infty & 5 & 0 & 5.875 & \infty & \infty & \infty & \infty & \infty & \infty \\
11.465 & \infty & \infty & \infty & \infty & 60.479 & 0 & 11 & \infty & \infty & \infty & \infty & \infty \\
\infty & \infty & \infty & \infty & \infty & \infty & 11 & 0 & 3.375 & \infty & \infty & \infty & 14.058 \\
\infty & \infty & \infty & \infty & \infty & \infty & \infty & 3.375 & 0 & 5 & \infty & \infty & \infty \\
\infty & \infty & \infty & \infty & \infty & \infty & \infty & \infty & 5 & 0 & 8.375 & \infty & \infty \\
\infty & \infty & \infty & 11 & \infty & \infty & \infty & \infty & \infty & 8.375 & 0 & 12.656 & \infty \\
\infty & \infty & \infty & \infty & \infty & \infty & \infty & \infty & \infty & \infty & 12.686 & 0 & 10.054 \\
\infty & \infty & \infty & \infty & \infty & \infty & \infty & 14.058 & \infty & \infty & \infty & 10.054 & 0
\end{array}\right]
$$

(8) Bring the optimized adjacency matrix into the Dijkstra algorithm for calculation, and obtain a new driving route $1 \rightarrow 2 \rightarrow 3 \rightarrow 4 \rightarrow 5 \rightarrow 6$. There are not any other influencing factors in the new route, so this new route is the optimal route from the entrance of the parking lot to the parking space.

\subsection{Simulation Analysis}

In the target parking lot scene, three kinds of parking lot conditions with different dynamic factors are selected to do the simulation. The test vehicle speed is set to $5 \mathrm{~km} / \mathrm{h}$.

Scene 1:

As shown in Figure 9, there are two cars going on the automatic parking. The remaining time is $45 \mathrm{~s}$ and $20 \mathrm{~s}$. In addition, there are two cars in the parking lot driving to follow the red lines to the parking spaces pointed by the red arrows to start automatic parking.

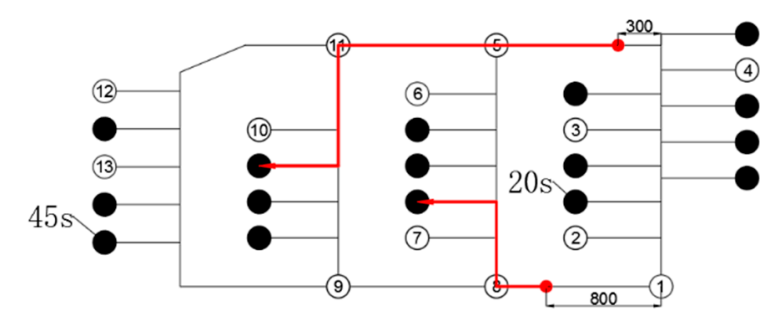

Figure 9. Real-time condition 1 of the parking lot.

The initial driving route of each parking space is shown in Table 2.

Table 2. Initial driving route of each parking space.

\begin{tabular}{cccc}
\hline Parking Space Number & Route & Distance/m & Time Consuming/s \\
\hline Parking Space 2 & $1 \rightarrow 2$ & 3.375 & 2.430 \\
Parking Space 3 & $1 \rightarrow 2 \rightarrow 3$ & 10.875 & 23.600 \\
Parking Space 4 & $1 \rightarrow 2 \rightarrow 3 \rightarrow 4$ & 15.025 & 26.588 \\
Parking Space 6 & $1 \rightarrow 8 \rightarrow 7 \rightarrow 6$ & 24.840 & 62.125 \\
Parking Space 7 & $1 \rightarrow 8 \rightarrow 7$ & 14.840 & 10.685 \\
Parking Space 10 & $1 \rightarrow 8 \rightarrow 9 \rightarrow 10$ & 33.340 & 71.845 \\
Parking Space 12 & $1 \rightarrow 8 \rightarrow 9 \rightarrow 13 \rightarrow 12$ & 47.027 & 52.563 \\
Parking Space 13 & $1 \rightarrow 8 \rightarrow 9 \rightarrow 13$ & 41.775 & 48.781 \\
\hline
\end{tabular}

The driving route of each parking space under the improved algorithm is shown in Table 3. 
Table 3. Driving route of each parking space under the improved algorithm.

\begin{tabular}{cccc}
\hline Parking Space Number & Route & Distance/m & Time Consuming/s \\
\hline Parking Space 2 & $1 \rightarrow 2$ & 3.375 & 2.430 \\
Parking Space 3 & $1 \rightarrow 2 \rightarrow 3$ & 10.875 & 23.600 \\
Parking Space 4 & $1 \rightarrow 2 \rightarrow 3 \rightarrow 4$ & 15.025 & 26.588 \\
Parking Space 6 & $1 \rightarrow 2 \rightarrow 3 \rightarrow 4 \rightarrow 5 \rightarrow 6$ & 24.840 & 62.125 \\
Parking Space 7 & $1 \rightarrow 8 \rightarrow 7$ & 14.840 & 10.685 \\
Parking Space 10 & $1 \rightarrow 2 \rightarrow 3 \rightarrow 4 \rightarrow 5 \rightarrow 11 \rightarrow 10$ & 33.340 & 71.845 \\
Parking Space 12 & $1 \rightarrow 8 \rightarrow 9 \rightarrow 13 \rightarrow 12$ & 47.027 & 52.563 \\
Parking Space 13 & $1 \rightarrow 8 \rightarrow 9 \rightarrow 13$ & 41.775 & 48.781 \\
\hline
\end{tabular}

Under the improved planning scheme, the corresponding driving route of parking space No. 6 and parking space No. 10 has changed. The time saved is $23.610 \mathrm{~s}$ and $15.192 \mathrm{~s}$, respectively, and the saving time accounts for $38.004 \%$ and $21.146 \%$ of the original time, respectively;

Scene 2:

As shown in Figure 10, there are two cars going on the automatic parking. The remaining time for each parking process is $50 \mathrm{~s}$. There is also a car in the parking lot driving following the red line to the parking space pointed by the red arrow to start automatic parking.

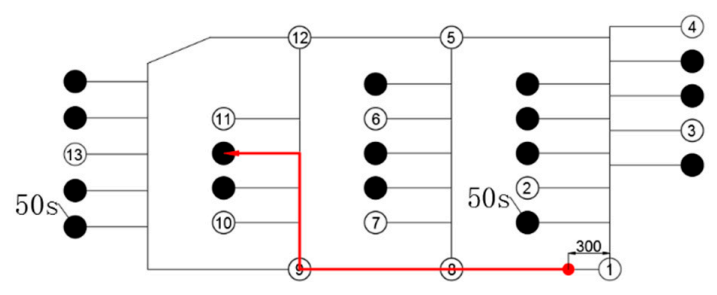

Figure 10. Real-time condition 2 of the parking lot.

The initial driving route of each parking space is shown in Table 4.

Table 4. Initial driving route of each parking space.

\begin{tabular}{cccc}
\hline Parking Space Number & Route & Distance/m & Time Consuming/s \\
\hline Parking Space 2 & $1 \rightarrow 2$ & 5.875 & 51.800 \\
Parking Space 3 & $1 \rightarrow 2 \rightarrow 3$ & 10.025 & 54.788 \\
Parking Space 4 & $1 \rightarrow 2 \rightarrow 3 \rightarrow 4$ & 17.525 & 60.188 \\
Parking Space 6 & $1 \rightarrow 8 \rightarrow 7 \rightarrow 6$ & 22.340 & 16.085 \\
Parking Space 7 & $1 \rightarrow 8 \rightarrow 7$ & 14.840 & 10.685 \\
Parking Space 10 & $1 \rightarrow 8 \rightarrow 9 \rightarrow 10$ & 25.840 & 18.605 \\
Parking Space 11 & $1 \rightarrow 8 \rightarrow 9 \rightarrow 10 \rightarrow 11$ & 33.340 & 71.845 \\
Parking Space 13 & $1 \rightarrow 8 \rightarrow 9 \rightarrow 13$ & 41.775 & 53.781 \\
\hline
\end{tabular}

The driving route of each parking space under the improved algorithm is shown in Table 5.

Table 5. Driving route of each parking space under the improved algorithm.

\begin{tabular}{cccc}
\hline Parking Space Number & Route & Distance/m & Time Consuming/s \\
\hline Parking Space 2 & $1 \rightarrow 8 \rightarrow 7 \rightarrow 6 \rightarrow 5 \rightarrow 3 \rightarrow 2$ & 50.555 & 36.400 \\
Parking Space 3 & $1 \rightarrow 8 \rightarrow 7 \rightarrow 6 \rightarrow 5 \rightarrow 3$ & 46.405 & 33.412 \\
Parking Space 4 & $1 \rightarrow 8 \rightarrow 7 \rightarrow 6 \rightarrow 5 \rightarrow 4$ & 40.455 & 29.128 \\
Parking Space 6 & $1 \rightarrow 8 \rightarrow 7 \rightarrow 6$ & 22.340 & 16.085 \\
Parking Space 7 & $1 \rightarrow 8 \rightarrow 7$ & 14.840 & 10.685 \\
Parking Space 10 & $1 \rightarrow 8 \rightarrow 9 \rightarrow 10$ & 25.840 & 18.605 \\
Parking Space 11 & $1 \rightarrow 8 \rightarrow 7 \rightarrow 6 \rightarrow 5 \rightarrow 12 \rightarrow 11$ & 45.090 & 32.465 \\
Parking Space 13 & $1 \rightarrow 8 \rightarrow 7 \rightarrow 6 \rightarrow 5 \rightarrow 12 \rightarrow 13$ & 57.153 & 41.150 \\
\hline
\end{tabular}


Under the improved planning scheme, the corresponding driving routes of parking spaces No. 2, No. 3, No. 4, No. 11 and No. 13 have changed. The time saved is $15.400 \mathrm{~s}, 21.376,31.060 \mathrm{~s}, 39.380 \mathrm{~s}$ and $12.631 \mathrm{~s}$, respectively, and the saving time accounts for $29.730 \%, 39.016 \%, 51.605 \%, 54.812 \%$ and $23.486 \%$ of the original time, respectively.

Scene 3:

As shown in Figure 11, there are two cars going on the automatic parking. The remaining time is $45 \mathrm{~s}$ and $50 \mathrm{~s}$. There is a car in the parking lot driving to follow the red line to the parking space pointed by the red arrow to start automatic parking.

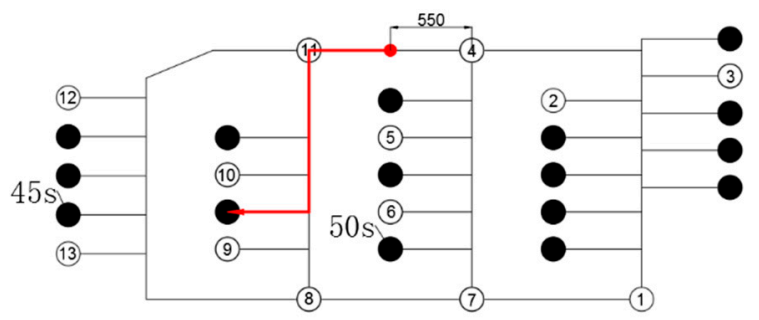

Figure 11. Real-time condition 3 of the parking lot.

The initial driving route of each parking space is shown in Table 6.

Table 6. Initial driving route of each parking space.

\begin{tabular}{cccc}
\hline Parking Space Number & Route & Distance/m & Time Consuming/s \\
\hline Parking Space 2 & $1 \rightarrow 2$ & 13.375 & 9.630 \\
Parking Space 3 & $1 \rightarrow 2 \rightarrow 3$ & 15.025 & 10.818 \\
Parking Space 5 & $1 \rightarrow 7 \rightarrow 6 \rightarrow 5$ & 22.340 & 55.400 \\
Parking Space 6 & $1 \rightarrow 7 \rightarrow 6$ & 17.340 & 51.800 \\
Parking Space 9 & $1 \rightarrow 7 \rightarrow 8 \rightarrow 9$ & 25.840 & 18.605 \\
Parking Space 10 & $1 \rightarrow 7 \rightarrow 8 \rightarrow 9 \rightarrow 10$ & 30.840 & 63.590 \\
Parking Space 12 & $1 \rightarrow 7 \rightarrow 8 \rightarrow 13 \rightarrow 12$ & 47.027 & 50.672 \\
Parking Space 13 & $1 \rightarrow 7 \rightarrow 8 \rightarrow 13$ & 36.523 & 26.297 \\
\hline
\end{tabular}

The driving route of each parking space under the improved algorithm is shown in Table 7.

Table 7. Driving route of each parking space under the improved algorithm.

\begin{tabular}{cccc}
\hline Parking Space Number & Route & Distance/m & Time Consuming/s \\
\hline Parking Space 2 & $1 \rightarrow 2$ & 13.375 & 9.630 \\
Parking Space 3 & $1 \rightarrow 2 \rightarrow 3$ & 15.025 & 10.818 \\
Parking Space 5 & $1 \rightarrow 2 \rightarrow 3 \rightarrow 4 \rightarrow 5$ & 34.090 & 24.545 \\
Parking Space 6 & $1 \rightarrow 2 \rightarrow 3 \rightarrow 4 \rightarrow 5 \rightarrow 6$ & 39.090 & 28.145 \\
Parking Space 9 & $1 \rightarrow 7 \rightarrow 8 \rightarrow 9$ & 25.840 & 18.605 \\
Parking Space 10 & $1 \rightarrow 2 \rightarrow 3 \rightarrow 4 \rightarrow 11 \rightarrow 10$ & 47.590 & 34.265 \\
Parking Space 12 & $1 \rightarrow 2 \rightarrow 3 \rightarrow 4 \rightarrow 11 \rightarrow 12$ & 51.901 & 37.369 \\
Parking Space 13 & $1 \rightarrow 7 \rightarrow 8 \rightarrow 13$ & 36.523 & 26.297 \\
\hline
\end{tabular}

Under the improved planning scheme, the corresponding driving routes of parking spaces No. 5, No. 6, No. 10 and No. 12 have changed. The time saved is 30.855 s, 23.655 s, 29.325 s and 13.303 s, respectively, and the saving time accounts for $55.695 \%, 45.666 \%, 46.116 \%$ and $26.253 \%$ of the original time, respectively.

\subsection{Summary}

Under the three conditions of the target parking lot, the improved parking space planning scheme is compared with the parking space guiding scheme based on the Dijkstra algorithm. The guiding 
routes of some parking spaces do not simply select the route with the shortest length. After taking the dynamic influencing factors into account in the parking lot, the route which takes less time to reach the target parking space is selected.

It means that the study arranges a new way for vehicles. It takes less time to reach their parking spaces. Vehicles will not gather in some short routes without considering the waiting time. It has become a great help in reducing the congestion in the parking lot.

\section{Conclusions and Future Research}

Because of the appearance of the valet parking technology, people do not need to find the parking space on their own in the complex parking lot environment. The technology also can solve the parking problem for some drivers if they have trouble parking because the vehicle is controlled by the system. The parking lot does not need to prepare much driving space for drivers with poor driving skills, because the driving and parking processes are all controlled by the system. The land use of the parking area will be much more rational.

The combination of the V2X environment and driverless technology is a new attempt in the parking assistance area. It has been a great help in making sure of the real-time condition and predicting future conditions in the parking lot. In this new technology environment, the base station can obtain the current working conditions of the parking lot in real-time. The vehicles in the valet parking lot are all driverless vehicles and the parking spaces are all standard. The speed can also be specified by the base station. These provide the data foundation for the planning scheme of this article.

Under the planning scheme of this paper, the driverless vehicle can avoid the congested roads in the valet parking lot and select the fastest route to reach the target parking space. It can reduce the congestion inside the parking lot.

For future research, more dynamic influence factors will be taken into consideration. The methods to deal with various conditions of the obstacle avoidance need to be found. To make the system have a wider applicability, the mixed condition of driverless vehicles and human drive vehicles should be considered. Overall, this study is an important step in the automatic valet parking technology. The following research will also have great help in the development of this technology.

Author Contributions: L.Y. and H.J. designed the scheme. L.H. checked the feasibility of the scheme. L.Y. performed the software simulation. L.Y. and L.H. analyzed the data of the simulation. L.Y. wrote the paper with the help of H.J. and L.H.

Funding: This work is financially supported by The Major University Nature Science Research Project of Jiangsu Province (No.16KJA580001).

Conflicts of Interest: The authors declare no conflict of interest.

\section{References}

1. Heimberger, M.; Horgan, J.; Hughes, C. Computer vision in automated parking systems: Design, implementation and challenges. Image Vis. Comput. 2017, 68, 88-101. [CrossRef]

2. Al-Turjman, F.; Malekloo, A. Smart parking in IoT-enabled cities: A survey. Sustain. Cities Soc. 2019, 49, 101608. [CrossRef]

3. Li, C.X. Research on Automatic Vertical Parking System Based on Intelligent Recognition Technology of Parking Space Scene. Ph.D. Thesis, Jiangsu University, Zhenjiang, China, 2016.

4. Ye, H.; Jiang, H.B.; Ma, S.D. Linear model predictive control of automatic parking path tracking with soft constraints. Int. J. Adv. Robot. Syst. 2019. [CrossRef]

5. De la Torre, G.; Rad, P.; Choo, K.K.R. Driverless vehicle security: Challenges and future research opportunities. Future Gener. Comput. Syst. 2018. [CrossRef]

6. Kaur, K.; Rampersad, G. Trust in driverless cars: Investigating key factors influencing the adoption of driverless cars. J. Eng. Technol. Manag. 2018, 48, 87-96. [CrossRef]

7. Zakharenko, R. Self-driving cars will change cities. Reg. Sci. Urban Econ. 2016, 61, 26-37. [CrossRef]

8. Pawel, G.; Inga, R. Traffic models for self-driving connected cars. Transp. Res. Procedia 2016, 14, 2207-2216. 
9. Enayattabar, M.; Ebrahimnejad, A.; Motameni, H. Dijkstra algorithm for shortest path problem under interval-valued Pythagorean fuzzy environment. Complex Intell. Syst. 2019, 5, 93-100. [CrossRef]

10. Bento, L.M.S.; Boccardo, D.R.; Machado, R.C.S.; Szwarcfiter, J.L. Dijkstra graphs. Discret. Appl. Math. 2019, 261, 52-62. [CrossRef]

11. Deng, Y.; Chen, Y.; Zhang, Y. Fuzzy Dijkstra algorithm for shortest path problem under uncertain environment. Appl. Soft Comput. 2012, 12, 1231-1237. [CrossRef]

12. Wang, S.X. The improved Dijkstra's shortest path algorithm and its application. Procedia Eng. 2012, 29, 1186-1190.

13. Balado, J.; Diaz-Vilarino, L.; Arias, P. Point clouds for direct pedestrian pathfinding in urban environments. ISPRS J. Photogramm. 2019, 148, 184-196. [CrossRef]

14. Binh, H.T.T.; Thanh, P.D.; Thang, T.B. New approach to solving the clustered shortest-path tree problem based on reducing the search space of evolutionary algorithm. Knowl. Based Syst. 2019, 180, 12-25. [CrossRef]

15. Xu, B.; Chen, X.L.; Li, K.Q. Double-layer speed optimization for reducing fuel consumption with vehicle-to-infrastructure communication. J. Intell. Transp. Syst. 2019, 23, 513-524. [CrossRef]

16. Shakhovska, N.; Shakhovska, K.; Fedushko, S. Some aspects of the method for tourist route creation. In Proceedings of the International Conference of Artificial Intelligence, Medical Engineering, Education (AIMEE), Moscow, Russia, 6-8 October 2018.

17. Yu, J.; Ge, X.X. Optimal route planning of parking lot based on Dijkstra algorithm. In Proceedings of the International Conference on Robots \& Intelligent System (ICRIS), Huai'an, China, 15-16 October 2017.

18. Yuan, L.Y.; Huang, R.F.; Han, L.J.; Zhou, M.M. A parking guidance algorithm based on time-optimal dynamic sorting for underground parking. In Proceedings of the 26th International Conference on Geoinformatics, Kunming, China, 28-30 June 2018.

19. Huang, C.M.; Chiang, M.S.; Dao, D.T. Vehicle-to-infrastructure (V2I) offloading from cellular network to 802.11p Wi-Fi network based on the software-defined network (SDN) architecture. Veh. Commun. 2017, 9, 288-300. [CrossRef]

20. Campolo, C.; Molinaro, A.; Iera, A. A reference framework for social-enhanced Vehicle-to-Everything communications in 5G scenarios. Comput. Netw. 2018, 143, 140-152. [CrossRef]

21. Dey, K.C.; Rayamajhi, A.; Chowdhury, M. Vehicle-to-vehicle (V2V) and vehicle-to-infrastructure (V2I) communication in a heterogeneous wireless network-Performance valuation. Transp. Res. Part C Emerg. Technol. 2016, 68, 168-184. [CrossRef]

22. Kunst, R.; Avila, L.; Binotto, A. Improving devices communication in Industry 4.0 wireless networks. Eng. Appl. Artif. Intell. 2019, 83, 1-12. [CrossRef]

23. Fernando, M.S. New option for real estate development "robot and cyborg parking system". The first national architectural research day. Madrid, Spain. 2007.

24. Liu, Q.; Chen, H.; Liu, Y. Model of the large parking lots guidance information system. In Proceedings of the 3rd International Conference on Transportation Engineering (ICTE), Chengdu, China, 23-25 July 2011.

25. Wang, G.Q.; Nakanishi, T.; Fukuda, A. Time-varying shortest path algorithm with transit time tuning for parking lot navigation. In Proceedings of the IEEE Region 10 Conference, Macao, China, 1-4 November 2015.

26. Tu, J.F. Parking lot guiding with IoT way. Microelectron. Reliab. 2019, 94, 19-23. [CrossRef]

27. Shin, J.H.; Jun, H.B. A study on smart parking guidance algorithm. Transp. Res. Part C Emerg. Technol. 2014, 44, 299-317. [CrossRef]

28. Wang, J.L.; Huang, H.; Qian, X.S. Sequence recognition of Chinese license plates. Neurocomputing 2018, 317, 149-158. [CrossRef]

29. Bjorklund, T.; Fiandrotti, A.; Annarumma, M. Robust license plate recognition using neural networks trained on synthetic images. Pattern Recognit. 2019, 93, 134-146. [CrossRef]

30. Spichkova, M.; Simic, M.; Schmidt, H. Formal model for intelligent route planning. Procedia Comput. Sci. 2015, 60, 1299-1308. [CrossRef]

31. Yanling, W.; Xin, W.; Ming, C.Z. Current situation and analysis of parking problem in Beijing. Procedia Eng. 2016, 137, 777-785. [CrossRef]

32. Sung, K.; Choi, J.; Kwak, D. Vehicle control system for automatic valet parking with infrastructure sensors. In Proceedings of the 2011 IEEE International Conference on Consumer Electronics (ICCE), Las Vegas, NV, USA, 9-12 January 2011; pp. 567-568. 
33. Li, M.; Chen, Y.; Zhou, A.J. Adaptive tracking control for networked control systems of intelligent vehicle. Inf. Sci. 2019, 503, 493-507. [CrossRef]

34. To, C.N.; Marzbani, H.; Simic, M. Auto driver autonomous vehicles control strategy. Procedia Comput. Sci. 2018, 126, 870-877. [CrossRef]

35. Zhao, J.X.; Xu, H.; Liu, H.C. Detection and tracking of pedestrians and vehicles using roadside LiDAR sensors. Transp. Res. Part C Emerg. Technol. 2019, 100, 68-87. [CrossRef]

36. Liang, W.; Long, J.; Lei, X. Efficient data packet transmission algorithm for IPV6 mobile vehicle network based on fast switching model with time difference. Future Gener. Comput. Syst. 2019, 100, 132-143. [CrossRef]

37. Quynh, L.K.; Tu, B.D.; Thuy, N.T. Meander anisotropic magnetoresistance bridge geomagnetic sensors. J. Sci. Adv. Mater. Devices 2019, 4, 327-332. [CrossRef]

38. Jiang, H.B.; Ye, H.; Ma, S.D. Method for accurately identifying parking space of automatic parking system based on multi-sensor data fusion. J. Chongqing Univ. Technol. 2019, 33, 1-10.

(C) 2019 by the authors. Licensee MDPI, Basel, Switzerland. This article is an open access article distributed under the terms and conditions of the Creative Commons Attribution (CC BY) license (http://creativecommons.org/licenses/by/4.0/). 